\title{
A DISCRETE TIME BENCHMARK APPROACH FOR INSURANCE AND FINANCE
}

\author{
HANs BÜHLmanN ${ }^{1}$ AND EcKhard Platen ${ }^{2}$
}

\begin{abstract}
This paper proposes a consistent approach to discrete time valuation in insurance and finance. This approach uses the growth optimal portfolio as reference unit or benchmark. When used as benchmark, it is shown that all benchmarked price processes are supermartingales. Benchmarked fair price processes are characterized as martingales. No measure transformation is needed for the fair pricing of insurance policies and derivatives. The standard actuarial pricing rule is obtained as a particular case of fair pricing when the contingent claim is independent from the growth optimal portfolio.
\end{abstract}

1991 Mathematics Subject Classification: primary 90A12; secondary 60G30, 62P20.

JEL Classification: G10, G13

\section{KEYWORDS AND PHRASES}

Financial and insurance market model, benchmark approach, growth optimal portfolio, numeraire portfolio, fair pricing, actuarial pricing, unit linked insurance.

\section{INTRODUCTION}

There exists a stream of literature that exploits the concept of a growth optimal portfolio (GOP), originally developed by Kelly (1956) and later extended and discussed, for instance, in Long (1990), Artzner (1997), Bajeux-Besnainou \& Portait (1997), Karatzas \& Shreve (1998), Kramkov \& Schachermayer (1999), Korn (2001) and Goll \& Kallsen (2002). Under certain assumptions the GOP coincides with the numeraire portfolio, which makes prices, when expressed in units of this particular portfolio, into martingales under the given probability

Department of Mathematics, Eidgenössische Technische Hochschule, 8092 Zürich, Switzerland.

2 University of Technology Sydney, School of Finance \& Economics and Department of Mathematical Sciences, PO Box 123, Broadway, NSW, 2007, Australia. 
measure. In Kramkov \& Schachermayer (1999) and Platen $(2001,2002)$ it was demonstrated that prices when benchmarked by the GOP can become supermartingales. The notion of a numeraire portfolio was recently extended by Becherer (2001), taking into account benchmarked prices that are supermartingales when an equivalent local martingale measure exists. In standard cases with an equivalent martingale measure the numeraire portfolio has been shown to coincide with the inverse of the deflator or state price density, see Constatinides (1992), Duffie (1996) or Rogers (1997). Furthermore, in Bühlmann $(1992,1995)$ and Bühlmann et al. (1998) the deflator has been suggested for the modeling of financial and insurance markets. Similarly, in Platen (2001, 2002, 2004) a financial market has been constructed by characterization of the GOP as benchmark portfolio.

Within this paper we follow a discrete time benchmark approach, where we characterize key features of a financial and insurance market via the GOP. We do not assume the existence of an equivalent martingale measure. The concept of fair pricing is introduced, where fair prices of insurance policies and derivatives are obtained via conditional expectations with respect to the real world probability measure. This provides a consistent basis for pricing that is widely applicable in insurance but also in derivative pricing. Examples of a discrete time market will be given that illustrate some key features of the benchmark approach.

\section{Discrete Time Market}

Let us consider a discrete time market that is modeled on a given probability space $(\Omega, \mathcal{A}, P)$. Asset prices are assumed to change their values only at the given discrete times

$$
0 \leq t_{0}<t_{1}<\ldots<t_{n}<\infty
$$

for fixed $n \in\{0,1, \ldots\}$. The information available at time $t$ in this market is described by $\mathcal{A}_{t_{i}}$. In this paper we consider $d+1$ primary securities, $d \in\{1,2, \ldots\}$, which generate interest, dividend, coupon or other payments as income or loss, incurred from holding the respective asset. We denote by $S_{i}^{(j)}$ the nonnegative value at time $t_{i}$ of a primary security account. This account holds only units of the $j$ th security and all income is reinvested into this account. The 0th primary security account is the domestic savings account. According to the above description, the domestic savings account $S^{(0)}$ is then a roll-over short term bond account, where the interest payments are reinvested at each time step. If the $j$ th primary security is a share, then $S_{i}^{(j)}$ is the value at time $t_{i}$ of such shares including accrued dividends. Thus, the quantity $S_{i}^{(j)}$ represents the $j$ th cum-dividend share price at time $t_{i}$. We assume that

$$
S_{i}^{(j)}>0
$$

almost surely for all $j \in\{0,1, \ldots, d\}$. 
Now, we introduce the growth ratio $h_{i+1}^{(j)}$ of the $j$ th primary security account at time $t_{i+1}$ in the form

$$
h_{i+1}^{(j)}= \begin{cases}\frac{S_{i+1}^{(j)}}{S_{i}^{(j)}} & \text { for } \quad S_{i}^{(j)}>0 \\ 0 & \text { otherwise }\end{cases}
$$

for $i \in\{0,1, \ldots, n-1\}$ and $j \in\{0,1, \ldots, d\}$. Note that the return of $S^{(j)}$ at time $t_{i+1}$ equals $h_{i+1}^{(j)}-1$. In our context the concept of a growth ratio will be more convenient than that of a return. We assume that $h_{i+1}^{(j)}$ is $\mathcal{A}_{t_{i+1}}$-measurable and almost surely finite. The growth rate of the domestic savings account $S^{(0)}$ shall be strictly positive, that is

$$
h_{i+1}^{(0)}>0
$$

almost surely for all $i \in\{0,1, \ldots, n-1\}$ with $S_{0}^{(0)}=1$. We can express the price of the $j$ th primary security account at time $t_{i}$, that is usually the $j$ th cumdividend share price $S_{i}^{(j)}$, in the form

$$
S_{i}^{(j)}=S_{0}^{(j)} \prod_{l=1}^{i} h_{l}^{(j)}
$$

for $i \in\{0,1, \ldots, n\}$ and $j \in\{0,1, \ldots, d\}$. Note that due to assumptions (2.1) and (2.3) we have for the savings account

$$
S_{i}^{(0)}>0
$$

for all $i \in\{0,1, \ldots, n\}$.

In the given discrete time market it is possible to form self-financing portfolios containing the above primary security accounts, where the changes in the value of the portfolio are only due to changes in primary security accounts. Since we will only consider self-financing portfolios we omit in the following the word "self-financing". For the characterization of a strictly positive portfolio at time $t_{i}$ it is sufficient to describe the proportion $\pi_{i}^{(j)} \in(-\infty, \infty)$ of its value that at this time is invested in the $j$ th primary security account, $j \in\{0,1, \ldots, d\}$. Obviously, the proportions add to one, that is

$$
\sum_{j=0}^{d} \pi_{i}^{(j)}=1
$$

for all $i \in\{0,1, \ldots, d\}$. The vector process $\pi=\left\{\pi_{i}=\left(\pi_{i}^{(0)}, \pi_{i}^{(1)}, \ldots, \pi_{i}^{(d)}\right), i \in\right.$ $\{0,1, \ldots, n\}\}$ denotes the corresponding process of proportions. We assume that $\pi_{i}$ is $\mathcal{A}_{t_{i}}$-measurable, which means that the proportions at a given time do not depend on any future events. The value of the corresponding portfolio at 
time $t_{i}$ is denoted by $S_{i}^{(\pi)}$ and we write $S^{(\pi)}=\left\{S_{i}^{(\pi)}, i \in\{0,1, \ldots, n\}\right\}$. Obviously, we obtain the growth ratio $h_{l}^{(\pi)}$ of this portfolio at time $t_{l}$ in the form

$$
h_{l}^{(\pi)}=\sum_{j=0}^{d} \pi_{l-1}^{(j)} h_{l}^{(j)}
$$

for $l \in\{1,2, \ldots, n\}$, where its value at time $t_{i}$ is given by the expression

$$
S_{i}^{(\pi)}=S_{0}^{(\pi)} \prod_{l=1}^{i} h_{l}^{(\pi)}
$$

for $i \in\{0,1, \ldots, n\}$.

\section{Discrete Time Market of Finite Growth}

Let us denote by $\mathcal{V}$ the set of all strictly positive portfolio processes $S^{(\pi)}$. This means, for a portfolio process $S^{(\pi)} \in \mathcal{V}$ it holds $h_{i+1}^{(\pi)} \in(0, \infty)$ almost surely for all $i \in\{0,1, \ldots, n-1\}$. Due to (2.5) $\mathcal{V}$ is not empty. We define for a given portfolio process $S^{(\pi)} \in \mathcal{V}$ with corresponding process of proportions $\pi$ its growth rate $g_{i}^{(\pi)}$ at time $t_{i}$ by the conditional expectation

$$
g_{i}^{(\pi)}=E\left(\log \left(h_{i+1}^{(\pi)}\right) \mid \mathcal{A}_{t_{i}}\right)
$$

for all $i \in\{0,1, \ldots, n-1\}$. This allows us to introduce the optimal growth rate $\underline{g}_{i}$ at time $t_{i}$ as the supremum

$$
\underline{g}_{i}=\sup _{S^{(\pi)} \in \mathcal{V}} g_{i}^{(\pi)}
$$

for all $i \in\{0,1, \ldots, n-1\}$.

If the optimal growth rate could reach an infinite value, then the corresponding portfolio would have unlimited growth. We exclude such unrealistic behaviour by introducing the following natural condition.

Assumption 3.1 We assume that the given discrete time market is of finite growth, that is

$$
\max _{i \in\{0,1, \ldots, n-1\}} \underline{g}_{i}<\infty
$$

almost surely.

Furthermore, it is natural to assume that our discrete time market is such that a portfolio exists, which attains the optimal growth rate. 
Assumption 3.2 There exists a portfolio $S^{(\underline{\pi})} \in \mathcal{V}$ with corresponding process of proportions $\underline{\pi}$ and

$$
S_{0}^{(\pi)}=1
$$

such that

$$
g_{i}^{(\pi)}=\underline{g}_{i}
$$

and

$$
E\left(\frac{h_{i+1}^{(\pi)}}{h_{i+1}^{(\pi)}} \mid \mathcal{A}_{t_{i}}\right)<\infty
$$

for all $i \in\{0,1, \ldots, n-1\}$ and $S^{(\pi)} \in \mathcal{V}$. Such a portfolio is called $a$ growth optimal portfolio $(G O P)$.

Without conditions (3.3) and (3.5) there is no basis for considering GOPs. Also condition (3.6) is a very natural condition, which only assumes the integrability of ratios of growth rates and thus allows to form conditional expectations. There is an extremely wide range of models that satisfy the Assumptions 3.1 and 3.2. These cover most established discrete time models used in insurance and finance.

From the viewpoint of an investor, a growth optimal portfolio (GOP), can be interpreted as a best performing portfolio because there is no other strictly positive portfolio that in the long term can outperform its optimal growth rate. The GOP has also another remarkable property, which we derive in the following. Let us study the situation that an investor puts almost all of his wealth in a GOP $S^{(\pi)}$ and invests a vanishing small proportion $\theta \in\left(0, \frac{1}{2}\right)$ into an alternative portfolio $S^{(\pi)} \in \mathcal{V}$. We call the resulting portfolio the interpolated portfolio $V^{\theta, \pi, \underline{\pi}} \in \mathcal{V}$. It exhibits by (2.7) at time $t_{i+1}$ the growth ratio

$$
h_{i+1}^{\theta, \pi, \underline{\pi}}=\frac{V_{i+1}^{\theta, \pi, \underline{\pi}}}{V_{i}^{\theta, \pi, \underline{\pi}}}=\theta h_{i+1}^{(\pi)}+(1-\theta) h_{i+1}^{(\pi)}
$$

with corresponding growth rate

$$
g_{i}^{\theta, \pi, \underline{\pi}}=E\left(\log \left(h_{i+1}^{\theta, \pi, \pi}\right) \mid \mathcal{A}_{t_{i}}\right)
$$

for $i \in\{0,1, \ldots, n-1\}$. To study the rate of change in the growth rate of the interpolated portfolio let us define its derivative in the direction of the alternative portfolio $S^{(\pi)} \in \mathcal{V}$ at time $t_{i}$, that is the limit

$$
\left.\frac{\partial g_{i}^{\theta, \pi, \pi}}{\partial \theta}\right|_{\theta=0+}=\lim _{\theta \rightarrow 0+} \frac{1}{\theta}\left(g_{i}^{\theta, \pi, \underline{\pi}}-g_{i}^{(\bar{\pi})}\right)
$$


for $i \in\{0,1, \ldots, n-1\}$. We prove in Appendix A the following fundamental identity, which will give us access to the understanding of the central role of the GOP in pricing.

Theorem 3.3 For a portfolio $S^{(\pi)} \in \mathcal{V}$ and $i \in\{0,1, \ldots n-1\}$ the derivative of the growth rate of the interpolated portfolio at time $t_{i}$ equals

$$
\left.\frac{\partial g_{i}^{\theta, \pi, \pi}}{\partial \theta}\right|_{\theta=0+}=E\left(\frac{h_{i+1}^{(\pi)}}{h_{i+1}^{(\pi)}} \mid \mathcal{A}_{t_{i}}\right)-1 .
$$

One observes that from (3.9), (3.5) and (3.2), we must have by the optimality property of the GOP that

$$
\left.\partial g_{i}^{\theta, \pi, \underline{\pi}}\right|_{\theta=0+} \leq 0,
$$

which leads by the identity (3.10) directly to the following important result.

Corollary 3.4 A portfolio process $S^{(\underline{\pi})} \in \mathcal{V}$ is growth optimal if and only if all portfolios $S^{(\pi)} \in \mathcal{V}$, when expressed in units of $S^{(\underline{\pi})}$, are $(\mathcal{A}, P)$-supermartingales, that is

$$
E\left(\frac{h_{i+1}^{(\pi)}}{h_{i+1}^{(\pi)}} \mid \mathcal{A}_{t_{i}}\right) \leq 1
$$

for all $i \in\{0,1, \ldots, n-1\}$.

Corollary 3.4 reveals a fundamental property of the GOP. It says, all nonnegative securities, when expressed in units of the GOP are supermartingales. Note that we did not make any major assumptions on the given discrete time market. Under the additional assumption on the existence of an equivalent local martingale measure, a similar result has been obtained for semimartingale markets in Becherer (2001). Corollary 3.4 is proved without the explicit assumption on the existence of an equivalent risk neutral measure. The simple and direct proof of Theorem 3.3 in the Appendix avoids the technical machinery employed in Becherer (2001). In addition, our approach is constructive and the fundamental equation (3.10) can be used to establish further identities or inequalities in risk management.

Let us consider two nonnegative portfolios that are both growth optimal, see (3.5). According to Corollary 3.4 the first portfolio, when expressed in units of the second, must be a supermartingale. Additionally, by the same argument the second, expressed in units of the first, must be also a supermartingale. This can only be true if both processes are identical, which yields the following result.

Corollary 3.5 The value process of the GOP is unique. 
Note that the stated uniqueness of the GOP does not imply that its proportions $\underline{\pi}$ have to be unique.

\section{FAir Portfolios}

In what follows we call prices, which are expressed in units of the GOP, benchmarked prices and their growth ratios benchmarked growth ratios. The condition (3.6) guarantees the integrability of benchmarked growth ratios and prices The benchmarked price $\hat{S}_{i}^{(\pi)}$ at time $t_{i}$ of a portfolio $S^{(\pi)}$ is defined by the relation

$$
\hat{S}_{i}^{(\pi)}=\frac{S_{i}^{(\pi)}}{S_{i}^{(\pi)}}
$$

for all $i \in\{0,1, \ldots, n\}$. By Corollary 3.4, the benchmarked price of a strictly positive portfolio $S^{(\pi)} \in \mathcal{V}$ is a supermartingale, which means by (3.12), (4.1), and (2.8) that

$$
\hat{S}_{i}^{(\pi)} \geq E\left(\hat{S}_{k}^{(\pi)} \mid \mathcal{A}_{t_{i}}\right)
$$

for all $k \in\{0,1, \ldots, n\}$ and $i \in\{0,1, \ldots, k\}$.

In common actuarial and financial valuations in competitive, liquid markets a price is typically chosen such that seller and buyer have no systematic advantage or disadvantage. The problem of such a description is hidden in the fact that one must specify the reference unit or numeraire and the corresponding probability measure that both buyers and sellers use to calculate their expected payoff. If one chooses the real world measure as obvious probability measure, then one needs still to determine the reference unit. We know from Long (1990) that under certain conditions benchmarked prices are martingales. In markets with a corresponding equivalent risk neutral martingale measure this price corresponds to the risk neutral price. For this reason we choose in our more general setting the GOP as numeraire. By using the real world probability measure to form expectations and the GOP as numeraire it follows from Corollary 3.4, as shown in (4.2), that any strictly positive portfolio price, when expressed in units of the GOP, must be a supermartingale. This could give an advantage to the seller of the portfolio $S^{(\pi)}$ if the equality in (4.2) is a strict one. Its expected future benchmarked payoff is in such a case less than its present value. The only situation when buyers and sellers are equally treated is when the benchmarked price process $\hat{S}^{(\pi)}$ is an $(\underline{\mathcal{A}}, P)$ martingale, that means

$$
\hat{S}_{k}^{(\pi)}=E\left(\hat{S}_{k}^{(\pi)} \mid \mathscr{A}_{t_{i}}\right)
$$

for all $k \in\{0,1, \ldots, n\}$ and $i \in\{0,1, \ldots, k\}$. Equation (4.3) means that the actual benchmarked price $\hat{S}_{i}^{(\pi)}$ is the best forecast of its future benchmarked values. 
Equivalently to (4.3) we have by (2.8) for the corresponding portfolio process $S^{(\pi)}$ that

$$
E\left(\frac{h_{i+1}^{(\pi)}}{h_{i+1}^{(\pi)}} \mid \mathcal{A}_{t_{i}}\right)=1
$$

for all $i \in\{0,1, \ldots, n-1\}$. This leads us naturally to the concept of fair pricing, see also Platen (2002):

Definition 4.1 We call a value process $V=\left\{V_{k}, k \in\{0,1, \cdots, n\}\right\}$ fair if its benchmarked value $\hat{V}_{k}=\frac{V_{k}}{S_{k}^{(\mathcal{T})}}$ forms an $(\underline{\mathcal{A}}, P)$-martingale.

By Definition 4.1 and application of Theorem 3.3 we directly obtain the following interesting characterization of fair prices.

Corollary 4.2 A given portfolio process $S^{(\pi)}$ is fair if and only if

$$
\left.\frac{\partial g_{i}^{\theta, \pi, \pi}}{\partial \theta}\right|_{\theta=0+}=0
$$

for all $i \in\{0,1, \ldots, n-1\}$.

Intuitively, Corollary 4.2 expresses the fact that a portfolio is fair if the maximum that the growth rate of the corresponding interpolated portfolio attains, is a genuine maximum. This typically means that the GOP proportions must satisfy the usual first order conditions in the direction of the portfolio. This will happen if $S^{(\mathcal{\pi})}$ is in the interior of $\mathcal{V}$ as in this case the derivative at zero may be taken from both sides.

\section{A Two Asset Example}

To illustrate key features of the given discrete time benchmark approach, let us consider a simple example of a market with two primary security accounts. The two primary securities are the domestic currency, which is assumed to pay zero interest, and a stock that pays zero dividends. The savings account at time $t_{i}$ is here simply the constant $S_{i}^{(0)}=1$ for $i \in\{0,1, \ldots, n\}$. The stock price $S_{i}^{(1)}$ at time $t_{i}$ is given by the expression

$$
S_{i}^{(1)}=S_{0}^{(1)} \prod_{l=1}^{i} h_{l}^{(1)}
$$

for $i \in\{0,1, \ldots, n\}$. Here the growth ratio $h_{l}^{(1)} \in(0, \infty)$ at time $t_{l}$ is assumed to be a random variable that can reach values, which are arbitrarily close 
close to 0 and $\infty$. Since the GOP has always to be strictly positive we must have

$$
\underline{\pi}_{i}^{(1)} \in[0,1]
$$

for all $i \in\{0,1, \ldots, n\}$. By (2.6) the GOP proportions $\underline{\pi}_{i}^{(0)}$ and $\underline{\pi}_{i}^{(1)}$ are such that

$$
\underline{\pi}_{i}^{(0)}=1-\underline{\pi}_{i}^{(1)}
$$

for all $i \in\{0,1, \ldots, n\}$. Obviously, the set $\mathcal{V}$ of strictly positive, portfolios $S^{(\pi)}$ is then characterized by those portfolios with proportion $\pi_{i}^{(1)} \in[0,1]$ for all $i \in\{0,1, \ldots, n\}$. The growth rate $g_{i}^{(\pi)}$ at time $t_{i}$ for a portfolio $S^{(\pi)} \in \mathcal{V}$ is according to (3.1) given by the expression

$$
g_{i}^{(\pi)}=E\left(\log \left(1+\pi_{i}^{(1)}\left(h_{i+1}^{(1)}-1\right)\right) \mid \mathcal{A}_{t_{i}}\right)
$$

for all $i \in\{0,1, \ldots, n-1\}$. Let us now compute the optimal growth rate of this market, see (3.1). The first derivative of $g_{i}^{(\pi)}$ with respect to $\pi_{i}^{(1)}$ is

$$
\frac{\partial g_{i}^{(\pi)}}{\partial \pi_{i}^{(1)}}=E\left(\frac{h_{i+1}^{(1)}-1}{1+\pi_{i}^{(1)}\left(h_{i+1}^{(1)}-1\right)} \mid \mathcal{A}_{t_{i}}\right)
$$

and the second derivative has the form

$$
\frac{\partial^{2} g_{i}^{(\pi)}}{\partial\left(\pi_{i}^{(1)}\right)^{2}}=-E\left(\frac{\left(h_{i+1}^{(1)}-1\right)^{2}}{\left(1+\pi_{i}^{(1)}\left(h_{i+1}^{(1)}-1\right)\right)^{2}} \mid \mathcal{A}_{t_{i}}\right)
$$

for $i \in\{0,1, \ldots, n-1\}$. We note that the second derivative is always negative, which indicates that the growth rate has at most one maximum. However, this maximum may refer to a proportion that does not belong to the interval $[0,1]$. To clarify such a situation we compute with (5.5) the values

$$
\left.\frac{\partial g_{i}^{(\pi)}}{\partial \pi_{i}^{(1)}}\right|_{\pi_{i}^{(1)}=0}=E\left(h_{i+1}^{(1)} \mid \mathcal{A}_{t_{i}}\right)-1
$$

and

$$
\left.\frac{\partial g_{i}^{(\pi)}}{\partial \pi_{i}^{(1)}}\right|_{\pi_{i}^{(1)}=1}=1-E\left(\frac{1}{h_{i+1}^{(1)}} \mid \mathcal{A}_{t_{i}}\right)
$$
for $i \in\{0,1, \ldots, n-1\}$. Due to (5.6) the first derivative $\frac{\partial g_{i}^{(\pi)}}{\partial \pi_{i}^{(1)}}$ is decreasing for
$\pi_{i}^{(1)}$ increasing. If 


$$
E\left(\left(h_{i+1}^{(1)}\right)^{\lambda} \mid \mathcal{A}_{t_{i}}\right) \geq 1
$$

for both $\lambda=1$ and $\lambda=-1$, then (5.7) and (5.8) are of opposite sign and hence there exists some $\underline{\pi}_{i}^{(1)} \in[0,1]$ such that

$$
\left.\frac{\partial g_{i}^{(\pi)}}{\partial \pi_{i}^{(1)}}\right|_{\pi_{i}^{(1)}=\pi_{i}^{(1)}}=0
$$

for $i \in\{0,1, \ldots, n-1\}$. Otherwise, if condition (5.9) is violated, then the optimal proportion is to be chosen at one of the boundary points. In this case the derivative (5.5) will not be zero at the optimal proportion and we obtain not a genuine maximum for the optimal proportion.

To check whether particular primary securities and portfolios are fair we now specify in our example the distribution of the growth ratios. Let us consider the case when the growth ratio $h_{i}^{(1)}$ is independent of the past and lognormally distributed such that

$$
\log \left(h_{i}^{(1)}\right) \sim \mathcal{N}\left(\mu \Delta, \sigma^{2} \Delta\right)
$$

with mean $\mu \Delta$, variance $\sigma^{2} \Delta>0$ and time step size $\Delta=t_{i+1}-t_{i}>0$.

1. At first, we clarify when the derivative $\frac{\partial g_{i}^{(\pi)}}{\partial \pi_{i}^{(1)}}$ can become zero for $\pi_{i}^{(1)} \in[0,1]$.
Because of

$$
E\left(\left(h_{i+1}^{(1)}\right)^{\lambda} \mid \mathcal{A}_{t_{i}}\right)=\exp \left\{\left(\lambda \mu+\frac{\sigma^{2}}{2}\right) \Delta\right\}
$$

for $\lambda=1$ and $\lambda=-1,(5.10)$ can only hold for $|\mu| \leq \frac{\sigma^{2}}{2}$. In this case it is also possible to show for $\Delta \rightarrow 0$ that the optimal proportion $\underline{\pi}_{i}^{(1)}$ for the GOP reaches asymptotically the value

$$
\lim _{\Delta \rightarrow 0^{-}} \pi_{i}^{(1)}=\frac{1}{2}+\frac{\mu}{\sigma^{2}},
$$

with limits

$$
\lim _{\Delta \rightarrow 0} E\left(\frac{h_{i+1}^{(0)}}{h_{i+1}^{(\tilde{x})}} \mid \mathcal{A}_{t_{i}}\right)=1
$$

and

$$
\lim _{\Delta \rightarrow 0} E\left(\frac{h_{i+1}^{(1)}}{h_{i+1}^{(\mathcal{( \tau )}}} \mid \mathcal{A}_{t_{i}}\right)=1
$$


and it follows for all strictly positive portfolios $S^{(\pi)} \in \mathcal{V}$ that

$$
\left.\frac{\partial g_{i}^{\theta, \pi, \pi}}{\partial \theta}\right|_{\theta=0}=0
$$

for $i \in\{0,1, \ldots, n-1\}$. Thus by Corollary 4.2 all portfolios $S^{(\pi)} \in \mathcal{V}$ are fair if the absolute mean to variance ratio is less than $\frac{1}{2}$, that is $\frac{|\mu|}{\sigma^{2}} \leq \frac{1}{2}$. This means, for all strictly positive benchmarked portfolios the expected logreturn of $S^{(1)}$ is not allowed to be greater than half of its squared variance.

2. In the case $\frac{\mu}{\sigma^{2}}<-\frac{1}{2}$, when the stock significantly underperforms, then the situation is different. The optimal proportion is

$$
\underline{\pi}_{i}^{(1)}=0
$$

for all $i \in\{0,1, \ldots, n-1\}$. For the GOP this requires to hold all investments in the savings account. Here we get

$$
E\left(\frac{h_{i+1}^{(1)}}{h_{i+1}^{(\pi)}} \mid \mathcal{A}_{t_{i}}\right)=\exp \left(\left(\mu+\frac{\sigma^{2}}{2}\right) \Delta\right)<1,
$$

which shows that the benchmarked stock price process $\hat{S}^{(1)}=\frac{S^{(1)}}{S^{(\pi)}}$ is a strict supermartingale and not a martingale. Thus $S^{(1)}$ is not fair according to Definition 4.1. Alternatively, we can check by Corollary 4.2 whether $S^{(1)}$ is fair. For the portfolio $\pi$ with all wealth invested in stock, that is $\pi_{i}=$ $\left(\pi_{i}^{(0)}, \pi_{i}^{(1)}\right)=(0,1)$, we obtain the derivative of the corresponding interpolated portfolio in the form

$$
\left.\frac{\partial g_{i}^{\theta, \pi, \pi}}{\partial \theta}\right|_{\theta=0+}=\exp \left(\left(\mu+\frac{\sigma^{2}}{2}\right) \Delta\right)-1<0,
$$

which shows by Corollary 4.1 that $S^{(1)}$ is not fair. On the other hand $\hat{S}^{(0)}$ is clearly a martingale and thus fair.

3. For $\frac{\mu}{\sigma^{2}}>\frac{1}{2}$ the stock is performing extremely well. The optimal propotion is

$$
\underline{\pi}_{i}^{(1)}=1
$$

for $i \in\{0,1, \ldots, n-1\}$. This means, for sufficiently large mean of the logarithm of the growth ratio of the stock one has to hold for the GOP all investments in the stock. In this case we get 


$$
E\left(\frac{h_{i+1}^{(0)}}{h_{i+1}^{(\pi)}} \mid \mathcal{A}_{t_{i}}\right)=\exp \left(\left(-\mu+\frac{\sigma^{2}}{2}\right) \Delta\right)<1,
$$

which says that the benchmarked domestic savings account $\hat{S}^{(0)}=\frac{S^{(0)}}{\left.S^{(\pi)}\right)}$ is a strict supermartingale. This means that $\hat{S}^{(0)}$ is not a martingale and thus by Definition 4.1 not fair. However, note that $\hat{S}^{(1)}=1$ is a martingale. For $\pi_{i}=\left(\pi_{i}^{(0)}, \pi_{i}^{(1)}\right)=(1,0)$ we have then

$$
\left.\frac{\partial g_{i}^{\theta, \pi, \pi}}{\partial \theta}\right|_{\theta=0+}=\exp \left(\left(-\mu+\frac{\sigma^{2}}{2}\right) \Delta\right)-1<0 .
$$

This confirms also by Corollary 4.2 that $S^{(0)}$ is not fair.

This example demonstrates that benchmarked prices are not always martingales. However, these benchmarked prices become martingales if the corresponding derivative of the growth rate of the interpolated portfolio in the direction of the security is zero, as follows from Corollary 4.2. Furthermore, the given lognormal example indicates that discrete time markets with securities, where the mean to variance ratio of the excess log-return over the risk free rate exceeds one half, may not be fair.

\section{Fair Pricing of Contingent Claims}

Now, let us consider a contingent claim $H_{i}$, which is an $\mathcal{A}_{t_{i}}$-measurable, possibly negative payoff, expressed in units of the domestic currency and has to be paid at a maturity date $t_{i}, i \in\{1,2, \ldots, n\}$. Note that the claim $H_{i}$ is not only contingent on the information provided by the observed primary security accounts $S_{l}^{(j)}$ up until time $t_{i}, j \in\{0,1, \ldots, d\}, l \in\{0,1, \ldots, i\}$, but as well on additional information contained in $\mathcal{A}_{t_{i}}$ as, for instance, the occurrence of defaults or insured events. Following our previous discussion and Definition 4.1 we obtain directly the following formula for the fair price of a contingent claim.

Corollarly 6.1 The fair price $U_{k}^{\left(H_{i}\right)}$ at time $t_{k}$ for the contingent claim $H_{i}$ satisfies the fair pricing formula

$$
U_{k}^{\left(H_{i}\right)}=S_{k}^{(\pi)} E\left(\frac{H_{i}}{S_{i}^{(\pi)}} \mid \mathcal{A}_{t_{k}}\right),
$$

for $k \in\{0,1, \ldots, i\}$.

Obviously, by (4.10) all fair contingent claim prices have a corresponding benchmarked fair price of the type 


$$
\hat{U}_{k}^{\left(H_{i}\right)}=\frac{U_{k}^{\left(H_{i}\right)}}{S_{k}^{(\pi)}}
$$

for all $k \in\{0,1, \ldots, i\}, i \in\{0,1, \ldots, n\}$, where the process $\hat{U}^{\left(H_{i}\right)}=\left\{U_{k}^{\left(H_{i}\right)}, k \in\right.$ $\{0,1, \ldots, i\}\}$ forms an $(\mathcal{A}, P)$-martingale according to Definition 4 .1. The argument can be easily extended to sums of contingent claims with $\mathcal{A}$-adapted maturity dates. Note that all fair portfolios and fair contingent claim prices form a price system, where benchmarked prices are $(\mathcal{A}, P)$-martingales.

If there exists only one equivalent risk neutral martingale measure, then the pricing formula (6.1) is the standard risk neutral pricing formula, used in finance, see Platen $(2001,2002,2004)$. However note, in this paper we do not assume the existence of such an equivalent risk neutral martingale measure and consider a more general framework.

Formally, one can extend (6.1) also for assessing the accumulated value for cashflows that occurred in the past, that is for $k \in\{i+1, i+2, \ldots\}$. Then we obtain

$$
U_{k}^{\left(H_{i}\right)}=\frac{H_{i}}{S_{i}^{(\pi)}} S_{k}^{(\pi)}
$$

for $i \in\{0,1, \cdots\}$ and $k \in\{i+1, i+2, \ldots\}$. In (6.3) we express the with earnings accumulated $t_{k}$-value of the payment $H_{i}$ made at time $t_{i}$. This interpretation is important for insurance accounting as will be discussed below.

An important case arises when a contingent claim $H_{i}$ with maturity $t_{i}$ is independent of the value $S_{i}^{(\pi)}$ of the GOP. Then by using (6.1) its fair price at time $t_{k}$ is obtained by the formula

$$
U_{k}^{\left(H_{i}\right)}=E\left(H_{i} \mid \mathcal{A}_{t_{k}}\right) P_{k}^{i},
$$

where

$$
P_{k}^{i}=E\left(\frac{S_{k}^{(\pi)}}{S_{i}^{(\pi)}} \mid \mathcal{A}_{t_{k}}\right)
$$

is the fair value at time $t_{k}$ of the zero coupon bond with maturity $t_{i}$ for $k \in$ $\{0,1, \ldots, i\}, i \in\{0,1, \ldots, n\}$. The formula (6.4) reflects the classical actuarial pricing formula that has been applied by actuaries for centuries to project future cashflows into present values, though with an "artificial" not financial market oriented understanding of $P_{k}^{i}$. Thus it turns out that the actuarial pricing approach is in this particular case generalized by the fair pricing concept that we introduced above through Definition 4.1. Note, in this case the knowledge of the particular dynamics of the GOP is not necessary since the zero coupon bond $P_{k}^{i}$ carries the relevant information needed from the GOP. 


\section{Fair Pricing of Sequences of Cashflows}

For the pricing of an insurance policy the actuarial task is the valuation of a sequence of cashflows $X_{0}, X_{1}, \ldots, X_{n}$, which are paid at the times $t_{0}, t_{1}, \ldots, t_{n}$, respectively. After each payment, its value is invested by the insurance company in a strictly positive portfolio, characterized by a process of proportions $\pi$. Here we choose an arbitrary process of proportions $\pi$, representing the investment portfolio of the insurance company. The benchmarked fair price $\hat{Q}_{0}$ at time $t_{0}$ for the above sequence of cashflows is according to (6.2) given by the expression

$$
\hat{Q}_{0}=E\left(\sum_{k=0}^{n} \frac{X_{k}}{S_{k}^{(\pi)}} \mid \mathcal{A}_{t_{0}}\right) .
$$

It follows that the benchmarked fair value $\hat{Q}_{i}$ at time $t_{i}$ for $i \in\{0,1, \ldots, n-1\}$ of this sequence of cashflows equals the sum

$$
\hat{Q}_{i}=\hat{C}_{i}+\hat{R}_{i}
$$

for $i \in\{0,1, \ldots, n\}$. Here we obtain

$$
\hat{C}_{i}=\frac{1}{S_{i}^{(\pi)}} \sum_{k=0}^{i} X_{k} \prod_{l=k}^{i-1} h_{l+1}^{(\pi)}
$$

which expresses the benchmarked value of the already accumulated payments. Furthermore,

$$
\hat{R}_{i}=E\left(\sum_{k=1+1}^{n} \frac{X_{k}}{S_{k}^{(\pi)}} \mid \mathcal{A}_{t_{i}}\right)
$$

is the benchmarked fair price at time $t_{i}$ for the remaining payments, which is called the prospective reserve, see Bühlmann 95 . It is easy to check that the process $\hat{Q}=\left\{\hat{Q}_{i}, i \in\{0,1, \ldots, n\}\right\}$ forms an $(\mathcal{A}, P)$-martingale for all choices of $\pi$ by the insurance company. When expressed in units of the domestic currency, we have at time $t_{i}$ for the above sequence of cashflows the fair value

$$
Q_{i}=S_{i}^{(\pi)} \hat{Q}_{i}
$$

for all $i \in\{0,1, \ldots, n\}$.

The above result is important, for instance, for the fair pricing of life insurance policies. Each insurance carrier can choose its own process of proportions $\pi$ to invest the payments that arise. However, the GOP, which is needed to value the prospective reserve, must be the same for all insurance companies in the same market. Above we clarified the role of the GOP for pricing the prospective reserve. We point out that the above analysis says nothing about the performance and riskiness of different investment strategies that 
the insurance carrier can choose. The growth rate for the investment portfolio becomes optimal, if the proportions of the GOP are used. If the insurance company aims to maximize the growth rate of its investments, then the fair pricing of an insurance policy and the optimization of the investment portfolio both involve the GOP.

\section{Unit Linked InSURANCE CONTRACTS}

In the insurance context we look again at the cashflows $X_{0}, X_{1}, \ldots, X_{n}$ but assume a specific form for these random variables. Intuitively, they stand now for unit linked claims and premiums. Hence they can be of either sign. The cashflow at time $t_{i}$ is of the form

$$
X_{i}=D_{i} S_{i}^{(\pi)}
$$

for $i \in\{1,2, \ldots, n\}$. The payments are linked to some strictly positive reference portfolio $S^{(\pi)} \in \mathcal{V}$ with given proportions $\pi$. The insurance contract specifies the reference portfolio $S^{(\pi)}$ and the random variables $D_{i}$, which are contingent on the occurrence of insured events during the period $\left(t_{i-1}, t_{i}\right]$, for instance, death, disablement or accidents.

The standard actuarial technique treats such contracts by using the reference portfolio process $S^{(\pi)}$ as numeraire and then deals with the unit linked random variables $D_{0}, D_{1}, \ldots, D_{n}$ at interest rate zero. It is reasonable to assume that these random variables are $\mathcal{A}$-adapted and independent of the reference portfolio process $S^{(\pi)}$.

The standard actuarial value $W_{i}^{(\pi)}$ of the payment stream at time $t_{i}$ is determined by the accumulated payments $C_{i}^{(\pi)}$ and the properly defined liability or prospective reserve $r_{i}$. The standard actuarial methodology assumes that the insurer invests all accumulated payments in the reference portfolio $S^{(\pi)}$. Then one obtains for $W_{i}^{(\pi)}$, when expressed in units of the domestic currency, the expression

$$
W_{i}^{(\pi)}=C_{i}^{(\pi)}+r_{i}
$$

with accumulated payments

$$
C_{i}^{(\pi)}=S_{i}^{(\pi)} \sum_{k=1}^{i} D_{k}
$$

and the liability or actuarial prospective reserve

$$
r_{i}=S_{i}^{(\pi)} E\left(\sum_{k=i+1}^{n} D_{k} \mid \mathcal{A}_{t_{i}}\right)
$$

for $i \in\{0,1, \ldots, n\}$. Observe the difference between $W_{i}^{(\pi)}$ and $Q_{i}$ as defined in (7.5). Hence the standard actuarial pricing and fair pricing will, in general, 
lead to different results. As we have seen previously in (6.4) this is to be expected when the cashflows are not independent of the GOP.

The benchmarked value $\hat{W}_{i}^{(\pi)}=\frac{W_{i}^{(\pi)}}{S_{i}^{(\pi)}}$ at time $t_{i}$ for the cashflows of this unit linked insurance contract is then by (8.2) of the form

$$
\hat{W}_{i}^{(\pi)}=\frac{C_{i}^{(\pi)}+r_{i}}{S_{i}^{(\pi)}}
$$

for $i \in\{0,1, \ldots, n\}$. On the other hand, the benchmarked fair value $\hat{Q}_{i}^{(\pi)}$ at time $t_{i}$ of the cashflows of this contract is according to (7.1) - (7.5) given by the expression

$$
\hat{Q}_{i}^{(\pi)}=\frac{C_{i}^{(\pi)}+R_{i}}{S_{i}^{(\pi)}}
$$

with fair prospective reserve

$$
R_{i}=S_{i}^{(\pi)} E\left(\sum_{k=i+1}^{n} \frac{D_{k} S_{k}^{(\pi)}}{S_{k}^{(\pi)}} \mid \mathcal{A}_{t_{i}}\right)
$$

for $i \in\{0,1, \ldots, n\}$. Under the natural condition of nonnegative fair prospective reserves one can prove that the benchmarked fair prospective reserve is less or equal the actuarial prospective reserve. The proof of the following inequality relies on the supermartingale property of $\frac{S_{k}^{(\pi)}}{S_{k}^{(\pi)}}, k \in\{0,1, \ldots, n\}$ and is shown in
Appendix B.

\section{Lemma 8.1 If}

$$
E\left(\sum_{k=m+1}^{n} D_{k} \mid \mathcal{A}_{t_{m}}\right) \geq 0
$$

for all $m \in\{0,1, \ldots, n-1\}$, then

$$
R_{i} \leq r_{i}
$$

for all $i \in\{0,1, \ldots, m-1\}$.

As by (8.4) we have

$$
r_{m}=S_{m}^{(\pi)} E\left(\sum_{k=m+1}^{n} D_{k} \mid \mathcal{A}_{t_{m}}\right)
$$


the condition (8.8) of the lemma means that the insurance contract defines a cashflow whose actuarial prospective reserve never becomes negative. This is usually observed as a practical constraint, since insurance products that allow for negative reserves have many defects. From (8.5) and (8.6) we immediately have under condition (8.8) the inequality

$$
\hat{Q}_{i}^{(\pi)} \leq \hat{W}_{i}^{(\pi)}
$$

for $i \in\{0,1, \ldots, n\}$. Reverting to property (8.9) we observe that there is, in general, a nonnegative difference

$$
r_{i}-R_{i} \geq 0
$$

between the actuarial and the fair prospective reserve. This difference is a consequence of the classical actuarial price calculation leading to the prospective reserve $r_{i}$ in (8.4). Of course, the actuarial and the fair prospective reserve coincide if one uses the GOP as reference portfolio.

\section{CONCLUSION}

We have shown that the growth optimal portfolio plays a central role for pricing in finance and insurance markets. The concept of fair contingent claim pricing has been introduced. Fair price processes, when measured in units of the growth optimal portfolio, form martingales. For contingent claims that are independent of the growth optimal portfolio fair prices also coincide with the classical actuarial prices, however, in general, this is not the case.

\section{ACKNOWLEDGEMENT}

The second author likes to express his gratitude to ETH Zurich for its kind hospitality during several visits. Both authors thank Freddy Delbaen, Walter Schachermayer, Martin Schweizer and Albert Shiryaev for valuable suggestions for improvements of this work. The paper has gone through different versions. The referees have been very helpful to identify its weak spots. 


\section{A. ApPendiX}

\section{Proof of Theorem 3.3}

For $\theta \in\left(0, \frac{1}{2}\right)$ and $S^{(\pi)} \in \mathcal{V}$ we consider the interpolated portfolio $V^{\theta, \pi, \pi} \in \mathcal{V}$, that is with growth ratio

$$
h_{i+1}^{\theta, \pi, \pi}>0
$$

given in (3.7) for $i \in\{0,1, \ldots, n-1\}$. One can then show, using $\log (x) \leq x-1$ and (3.7), that

$$
G_{i+1}^{\theta, \pi, \underline{\pi}}=\frac{1}{\theta} \log \left(\frac{h_{i+1}^{\theta, \pi, \pi}}{h_{i+1}^{(\pi)}}\right) \leq \frac{1}{\theta}\left(\frac{h_{i+1}^{\theta, \pi, \pi}}{h_{i+1}^{(\tilde{\pi})}}-1\right)=\frac{h_{i+1}^{(\pi)}}{h_{i+1}^{(\pi)}}-1
$$

and

$$
G_{i+1}^{\theta, \pi, \underline{\pi}} \geq-\frac{1}{\theta}\left(\frac{h_{i+1}^{(\boldsymbol{\pi})}}{h_{i+1}^{\theta, \pi, \underline{\pi}}}-1\right)=\frac{h_{i+1}^{(\pi)}-h_{i+1}^{(\underline{\pi})}}{h_{i+1}^{\theta, \pi, \underline{\pi}}}
$$

We obtain in (A.3) for $h_{i+1}^{(\pi)}-h_{i+1}^{(\mathcal{T})} \geq 0$ because of $h_{i+1}^{\theta, \pi, \underline{\pi}}>0$ the inequality

$$
G_{i+1}^{\theta, \pi, \underline{\pi}} \geq 0
$$
and for $h_{i+1}^{(\pi)}-h_{i+1}^{(\pi)}<0$ from (A.3) because of $\theta \in\left(0, \frac{1}{2}\right), h_{i+1}^{(\pi)} \geq 0$, and $h_{i+1}^{\theta, \pi, \pi}>0$
that

$$
G_{i+1}^{\theta, \pi, \underline{\pi}} \geq-\frac{h_{i+1}^{(\tilde{\mu})}}{h_{i+1}^{\theta, \pi, \underline{\pi}}}=-\frac{1}{1-\theta+\theta \frac{h_{i+1}^{(\pi)}}{h_{i+1}^{(\tilde{\mu})}}} \geq-\frac{1}{1-\theta} \geq-2 .
$$

Summarizing (A.2)-(A.5) we have for $i \in\{0,1, \ldots, n-1\}$ and $S^{(\pi)} \in \mathcal{V}$ the upper and lower bounds

$$
-2 \leq G_{i+1}^{\theta, \pi, \underline{\pi}} \leq \frac{h_{i+1}^{(\pi)}}{h_{i+1}^{(\pi)}}-1,
$$

where by (3.6)

$$
E\left(\frac{h_{i+1}^{(\pi)}}{h_{i+1}^{(\mathcal{x})}}\right)<\infty
$$


Then by using (A.6) and (A.7) it follows by the Dominated Convergence Theorem that

$$
\begin{aligned}
\left.\frac{\partial g_{i}^{\theta, \pi, \underline{\pi}}}{\partial \theta}\right|_{\theta=0} & =\lim _{\theta \rightarrow 0+} E\left(G_{i+1}^{\theta, \pi, \pi} \mid \mathcal{A}_{t_{i}}\right) \\
& =E\left(\lim _{\theta \rightarrow 0+} G_{i+1}^{\theta, \pi, \pi} \mid \mathcal{A}_{t_{i}}\right) \\
& =E\left(\left.\frac{\partial}{\partial \theta} \log \left(\frac{h_{i+1}^{\theta, \pi, \pi}}{h_{i+1}^{(\pi)}}\right)\right|_{\theta=0} \mid \mathcal{A}_{t_{i}}\right) \\
& =E\left(\frac{h_{i+1}^{(\pi)}}{h_{i+1}^{(\pi)}} \mid \mathcal{A}_{t_{i}}\right)-1
\end{aligned}
$$

for $i \in\{0,1, \ldots, n-1\}$ and $S^{(\pi)} \in \mathcal{V}$. This proves equation (3.10).

$$
\text { B. APPENDIX }
$$

\section{Proof of Lemma 8.1}

We have

$$
E\left(\sum_{k=i+1}^{n} \frac{D_{k} S_{k}^{(\pi)}}{S_{k}^{(\pi)}} \mid \mathcal{A}_{t_{n-1}}\right) \leq E\left(\sum_{k=i+1}^{n} \frac{D_{k} S_{k \wedge(n-1)}^{(\pi)}}{S_{k \wedge(n-1)}^{(\pi)}} \mid \mathcal{A}_{t_{n-1}}\right)
$$

if $E\left(D_{n} \mid \mathcal{A}_{t_{n-1}}\right) \geq 0$,

$$
E\left(\sum_{k=i+1}^{n} \frac{D_{k} S_{k \wedge(n-1)}^{(\pi)}}{S_{k \wedge(n-1)}^{(\pi)}} \mid \mathcal{A}_{t_{n-2}}\right) \leq E\left(\sum_{k=i+1}^{n} \frac{D_{k} S_{k \wedge(n-2)}^{(\pi)}}{S_{k \wedge(n-2)}^{(\pi)}} \mid \mathcal{A}_{t_{n-2}}\right)
$$

if $E\left(D_{n}+D_{n-1} \mid \mathcal{A}_{t_{n-2}}\right) \geq 0$

$$
\begin{aligned}
E\left(\sum_{k=i+1}^{n} \frac{D_{k} S_{k \wedge(i+1)}^{(\pi)}}{S_{k \wedge(i+1)}^{(\pi)}} \mid \mathcal{A}_{t_{i}}\right) & \leq E\left(\sum_{k=i+1}^{n} \frac{D_{k} S_{k \wedge i}^{(\pi)}}{S_{k \wedge i}^{(\pi)}} \mid \mathcal{A}_{t_{i}}\right) \\
& =\frac{r_{i}}{S_{i}^{(\pi)}}
\end{aligned}
$$


if $E\left(D_{n}+D_{n-1}+\ldots+D_{i+1} \mid \mathcal{A}_{t}\right) \geq 0$, for $i \in\{0,1, \ldots, n-1\}$. Taking conditional expectation with respect to $\mathcal{A}_{t}$, the inequalities above become a chain, whose first member equals $\frac{R_{i}}{S_{i}^{(\pi)}}$, and the last member becomes $\frac{r_{i}}{S_{i}^{(\pi)}}$.

This proves (8.9).

\section{REFERENCES}

Artzner, P. (1997) On the numeraire portfolio. In Mathematics of Derivative Securities, pp. 5358. Cambridge University Press.

Bajeux-Besnainou, I. and Portait, R. (1997) The numeraire portfolio: A new perspective on financial theory. The European Journal of Finance 3, 291-309.

BECHERER, D. (2001) The numeraire portfolio for unbounded semimartingales. Finance Stoch. 5, 327-341.

BüHlmann, H. (1992) Stochastic discounting. Insurance: Mathematics and Economics 11, $113-$ 127.

BüHLManN, H. (1995) Life insurance with stochastic interest rates. In G. Ottaviani (Ed.), Financial Risk and Insurance, pp. 1-24. Springer.

Bühlmann, H., Delbaen, F., Embrechts, P. and Shiryaev, A. (1998) On Esscher transforms in discrete finance models. ASTIN Bulletin 28(2), 171-186.

Constatinides, G.M. (1992) A theory of the nominal structure of interest rates. Rev. Financial Studies 5, 531-552.

Duffie, D. (1996) Dynamic Asset Pricing Theory (2nd ed.). Princeton, University Press.

Goll, T. and KAlLSEN, J. (2003) A complete explicit solution to the log-optimal portfolio problem. Adv. in Appl. Probab. 13(2), 774-799.

Heath, D. and Platen, E. (2002) Pricing and hedging of index derivatives under an alternative asset price model with endogenous stochastic volatility. In J. Yong (Ed.), Recent Developments in Mathematical Finance, pp. 117-126. World Scientific.

Karatzas, I. and Shreve, S.E. (1998) Methods of Mathematical Finance, Volume 39 of Appl. Math. Springer.

Kelly, J.R. (1956) A new interpretation of information rate. Bell Syst. Techn. J. 35, 917-926.

KoRN, R. (2001) Value preserving strategies and a general framework for local approaches to optimal portfolios. Math. Finance 10(2), 227-241.

Kramkov, D.O. and Schachermayer, W. (1999) The asymptotic elasticity of utility functions and optimal investment in incomplete markets. Ann. Appl. Probab. 9, 904-950.

LonG, J.B. (1990) The numeraire portfolio. J. Financial Economics 26, 29-69.

Platen, E. (2001) A minimal financial market model. In Trends in Mathematics, pp. 293-301. Birkhäuser.

Platen, E. (2002) Arbitrage in continuous complete markets. Adv. in Appl. Probab. 34(3), 540558.

Platen, E. (2004). A class of complete benchmark models with intensity based jumps to appear in J. Appl. Probab. 4(1).

Rogers, L.C.G. (1997) The potential approach to the term structure of interest rates and their exchange rates. Math. Finance 7, 157-176. 